\title{
A giant chimeric NLR gene confers extreme broad- spectrum resistance to a plant pathogen
}

Jianxin Ma ( $\nabla$ maj@purdue.edu )

Purdue University https://orcid.org/0000-0002-1474-812X

Weidong Wang

Purdue University

Liyang Chen

Purdue University

Kevin Fengler

Corteva Agriscience

Joy Bolar

Corteva Agriscience

Victor Llaca

Corteva Agriscience https://orcid.org/0000-0003-4822-2924

Xutong Wang

Purdue University

Chancelor Clark

Purdue University

Tomara Fleury

USDA-ARS

Jon Myrvold

Corteva Agriscience

David Oneal

Corteva Agriscience

Daleen van Dyk

Corteva Agriscience

Ashly Hudson

Corteva Agriscience

Jesse Munkvold

Corteva Agriscience

Andy Baumgarten

Corteva Agriscience

Jeff Thompson

Corteva Agriscience

Guohong Cai 
USDA-ARS

\section{Oswald Crasta}

Equinom

\section{Rajat Aggarwal}

Corteva Agriscience

\section{Article}

Keywords: plant pathogens, chimeric NLR gene, resistance

Posted Date: July 12th, 2021

DOl: https://doi.org/10.21203/rs.3.rs-654548/v1

License: (c) (i) This work is licensed under a Creative Commons Attribution 4.0 International License. Read Full License

Version of Record: A version of this preprint was published at Nature Communications on November 5th, 2021. See the published version at https://doi.org/10.1038/s41467-021-26554-8. 
Title: A giant chimeric NLR gene confers extreme broad-spectrum resistance to a plant pathogen

2

3 Weidong Wang $^{1 *}$, Liyang Chen $^{1 *}$, Kevin Fengler $^{2}$, Joy Bolar ${ }^{2}$, Victor Llaca ${ }^{2}$, Xutong $4 \quad$ Wang $^{1}$, Chancelor B. Clark ${ }^{1}$, Tomara J Fleury ${ }^{3,4}$, Jon Myrvold ${ }^{2}$, David Oneal ${ }^{2}$, Maria Magdalena $5 \quad$ van Dyk ${ }^{2}$, Ashley Hudson ${ }^{2}$, Jesse Munkvold², Andy Baumgarten², Jeff Thompson ${ }^{2}$, Guohong

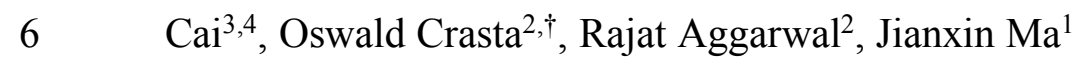

${ }^{1}$ Department of Agronomy, Purdue University, West Lafayette, IN 47907, USA.

${ }^{2}$ Research and Development, Corteva Agriscience ${ }^{\mathrm{TM}}$, Johnston, IA 50131, USA.

${ }^{3}$ Department of Botany and Plant Pathology, Purdue University, West Lafayette, IN 47907, USA.

${ }^{4}$ Crop Production and Pest Control Research Unit, USDA, ARS, West Lafayette, IN 47907, USA.

†Present address: R\&D, Equinom Inc., Indianapolis, IN 46268, USA.

3 *These authors contributed equally to this work.

Correspondence to J.M. (maj@purdue.edu) or R.A. (rajat.aggarwal@corteva.com) 
Abstract:

17 Phytophthora root and stem rot (PRSR) caused by Phytophthora sojae is the most destructive 18 soybean soil-borne disease worldwide. Discovery of genes conferring broad-spectrum 19 resistance to the pathogen is an urgent need to prevent the outbreak of the disease. Here we 20 show that soybean $R p s 11$ is a $27.7-\mathrm{kb}$ nucleotide-binding site-leucine-rich repeat (NBS-LRR 21 or NLR) gene conferring extreme broad-spectrum resistance to the pathogen. $R p s 11$ is

22 located in a genomic region harboring a cluster of unusually large NLR genes belonging to a 23 single evolutionary lineage that is distinct from all other lineages in the soybean genome, and 24 was derived from rounds of intergenic and intragenic unequal recombination. Such 25 recombination events have resulted in promoter fusion and expansion of the LRR domain 26 that presumably explains such broadness of the resistance spectrum. The NLR gene cluster 27 exhibits drastic structural diversification among phylogenetically representative varieties, 28 including gene copy number variation ranging from five to 23 copies, and absence of allelic 29 copies of $\operatorname{Rps} 11$ (i.e., rps11) in any of the non-Rps11-donor varieties examined. Our study 30 thus exemplifies innovative evolution of NLR genes and NLR gene clusters and will 31 accelerate the deployment of Rps11 for soybean protection.

33 Main

34 Phytophthora is a genus of plant-damaging oomycetes with more than 170 identified species $^{1}$, 35 many of which are plant pathogens of considerable economic importance such as Phytophthora 36 infestans that caused the Irish potato famine and subsequent diaspora ${ }^{2}$, and Phytophthora sojae 37 that is responsible for annual yield losses estimated at $\sim \$ 200$ million in the United States and 1-2 38 billion worldwide ${ }^{3,4}$. In general, plant diseases caused by Phytophthora are difficult to control 
39 chemically ${ }^{5}$, thus deployment of resistant varieties is an effective and environmentally friendly

40 strategy to manage Phytophthora diseases ${ }^{6}$. In recent decades, a few dozen resistance-to- $P$. sojae

41 (Rps) loci/alleles have been mapped to a number of genomic regions enriched with NLR genes

42 (soybease.org); nevertheless, many of those loci/alleles have become ineffective to newly evolved

43 races/isolates of the pathogen ${ }^{7}$, and few Rps loci/alleles have been isolated and/or functionally

44 validated. Therefore, effective introgression/pyramiding of specific Rps genes into elite soybean

45 cultivars for sustained resistance remains challenging.

47 The Rps11 locus alone is responsible for the extreme broad-spectrum resistance

48 We have recently identified an Rps locus, designated Rps11, in a soybean landrace, PI 594527,

49 which carries resistance to 12 P. sojae races/isolates examined ${ }^{8}$ (Extended Data Fig. 1). Based on

50 the responses of $209 \mathrm{~F}_{2: 3}$ families derived from a cross between PI 594527 and a susceptible variety

51 Williams to $P$. sojae race 1 , Rps11 was initially mapped to a $348-\mathrm{kb}$ genomic region on

52 chromosome $7^{8}$, with 12 sequencing gaps of unknown sizes according to the Williams 82 reference

53 genome assembly v2.0 (soybase.org). Nevertheless, the initial mapping only indicates that Rps 11

54 confers resistance to race 1 . To evaluate the Rps 11 resistance, we inoculated a subset of the $\mathrm{F}_{2: 3}$

55 families, including 14 families of the Rps11/Rps11 (PI 594527) genotype and 14 families of the

$56 r p s 11 / r p s 11$ (Williams) genotype with 14 P. sojae races/isolates separately, and observed perfect

57 genotype-phenotype associations (Supplemental Table1), suggesting that the Rps11 locus alone is

58 responsible for the broad spectrum of resistance carried by PI 594527. We then evaluated $\mathrm{F}_{5}$

59 recombinant inbred lines (RIL) derived from several $\mathrm{F}_{2}$ homozygotes (Rps11/Rps 11) with 158

60 additional P. sojae isolates collected from soybean fields across Indiana and found that those RILs

61 carry resistance to $127(80 \%)$ isolates (Supplemental Table 2). 


\section{Rps11 is pinpointed through gap-free sequence-based fine mapping with a sizable population}

64 Because the rps11 region in Williams 82, which is identical to the corresponding region in

65 Williams, had not been fully assembled, and also because this region is enriched with NLR genes

66 and thus thought to be highly labile, it was difficult to design markers for fine mapping. In order

67 to clone Rps11, we sequenced and assembled the PI 594527 genome into 43 scaffolds with a

68 scaffold N50 of $26.4 \mathrm{Mb}$ (see details in Method). In the course of PI 594527 sequencing, Chu et

69 al. developed a high-quality Williams 82 genome assembly ${ }^{9}$ (dubbed v3.0), which reveals a

70 number of assembly errors in the rps 11 region in the Williams 82 assembly v2.0 (Extended Data

71 Fig. 2). To eliminate potential inaccuracy in the initial Rps11 mapping result caused by those

72 assembly errors, we annotated and compared the Rps11/rps11 regions harboring the entire NLR

73 clusters from the PI 594527 genome assembly and the Williams 82 genome assembly v3.0. The

74 Rps11 region from PI 594527 is composed of 12 NLR genes (dubbed R1-R12) (Fig. 1a). Of these,

$75 \mathrm{R} 1, \mathrm{R} 4, \mathrm{R} 6, \mathrm{R} 7, \mathrm{R} 9, \mathrm{R} 11$, and R12 are intact and predicted to encode large NLR proteins containing

76 2,315-2,463 amino acids; R2, R3, R5 and R8 are truncated, with 2 to 4 exons lost at their 3' ends;

77 R10 carries a 1.4-kb insertion in its first exon (Extended Data Fig. 3a). Only R1, R4, R6, R9 and

78 R12 are expressed in stems inoculated with race 1 as revealed by RNA-seq (Extended Data Fig.

$793 \mathrm{~b})$. The corresponding region from the Williams 82 assembly v.3.0 is composed of eight NLR

80 genes (dubbed r1-r8) (Fig. 1a and Extended Data Fig. 4). Only a small proportion of the region is

81 shared between PI 594527 and Williams 82 as syntenic blocks, which allowed us to design markers

82 for fine mapping (Fig. 1a). 
84 Using two boundary markers, SSR286 and SSR320, in the conserved regions adjacent to the NLR

85 clusters, we genotyped 17,050 $\mathrm{F}_{4}$ plants derived from heterozygous (Rps11/rps 11) $\mathrm{F}_{3}$ plants of the

86 initial mapping population and identified 43 recombinants between the two markers (Extended

87 Data Fig. 5). These recombinants were then genotyped with eight additional markers distributed

88 in the syntenic blocks between the boundary markers and inoculated with P. sojae race 1, and

89 eventually defined the Rps 11 locus to a 151-kb region of PI 594527, harboring four NLR genes

90 (R5, R6, R7 and R8) (Fig. 1b and Extended Data Fig. 5). Of the four genes, only R6 is expressed

91 in inoculated stems and thus considered as the candidate gene of Rps 11 (Fig. 1c and Extended Data

92 Fig. 5). Consistently, only recombinants in which the expression of R6 was detected are resistant

93 to race 1 (Fig. 1c and Extended Data Fig. 6). R6 is also expressed in other tissues (Fig. 1d) and is

94 predicted to encode an NLR protein of 2,463 amino acids. The genomic DNA of R6, excluding its

95 regulatory sequences, is $14.1-\mathrm{kb}$, but it possesses a $13.1-\mathrm{kb} 5^{\prime}$ untranslated region (UTR) as

96 determined by 5' Rapid amplification of cDNA ends (5'RACE) (Fig. 1e) and a 0.5-kb 3'UTR,

97 totaling $27.7 \mathrm{~kb}$ (Fig. 1f).

98

99 The candidate gene for Rps11 is functionally validated through complementation test

100 To functionally validate the candidacy of R6 for Rps 11 , we expressed the coding sequence (CDS)

101 of R6 using a ubiquitin promoter in an elite soybean variety 93Y21, which is susceptible to $P$.

102 sojae races 25,31 and $\mathrm{OH} 12108-06-03$. As expected, the $\mathrm{T}_{2}$ progeny with the R6-transgene

103 showed resistance to these three races at levels similar to those shown by a homozygous $\mathrm{F}_{5}$ RIL

104 (Rps11/Rps11) derived from the cross between PI 594527 and Williams (Fig. 2a-b, and Extended

105 Data Fig. 7). By contrast, the non-transgenic 93Y21 (Control) was susceptible to these same races

106 (Fig. 2a-b and Extended Data Fig. 7). As expected, the expression of the R6-transgene was detected 
107 in those $\mathrm{T}_{2}$ progenies (Fig. 2c), and its expression level in each of the two transformation events is

108 correlated with the level of resistance as indicated by the survival rate of inoculated seedlings

109 (Extended Data Fig. 8). Together, these observations confirm that R6 in PI 594527 is Rps11.

111 Rps11 possesses a chimeric structure and lacks allelic copies in the soybean pangenome

112 In the PI 594527 genome assembly, a total of 512 NLR genes were annotated (Supplemental Table

1133 and Supplemental Table 4). Apart from the large NLR genes in the Rps 11 region, there are six

114 additional large NLR genes in the genome - one located on chromosome 16, and five clustered in

115 a region on chromosome 18. Compared with a typical NLR gene, the large genes were enlarged

116 by tandem duplications of the LRR domain (Fig. 3a). We found that all these large genes are

117 phylogenetically grouped into a single clade (Fig. 3b) and share similar structures (Extended Data

118 Fig. 3a and Extended Data Fig. 4), suggesting that they share a single origin. Comparative analysis

119 reveals that these three genomic regions hosting the large NLR genes were derived from two

120 soybean whole genome duplication (WGD) events ${ }^{10}$. Dot plot and phylogenetic analyses suggest

121 that the 12 NLR genes in the Rps 11 region were likely derived from a single copy R5, after the

122 second WGD event occurred $\sim 13$ million years ago, through rounds of tandem duplication events,

123 each involving one or two adjacent NLR genes (Fig. 3c-e). Apparently, the tandem duplications

124 were also accompanied by removal of NLR genes from the region, leading to the absence of allelic

125 copies of most NLR genes between PI 594527 and Williams 82/Williams.

127 Subsequently, we annotated 296 NLR genes in the corresponding regions from the high-quality 128 genome assemblies of 28 soybean accessions chosen to construct a pan-genome ${ }^{11-14}$, and observed 129 a striking copy number variation of NLR genes among these varieties, ranging from 5 to 23 copies 
130 (Fig. 4 and Supplemental Table 5). Such variation is primarily caused by unequal recombination

131 between different NLR genes, resulting in segmental duplications, deletions, and inversions (Fig.

132 4, Box 1 and Box 2). The variation appears to be associated with the phylogenetic relationships

133 among the accessions revealed by the SNP data within the Rps 11 region (Fig. 4). Inter-genomic

134 comparison demonstrates that Rps11 is a unique gene in PI 594527 without an allelic copy in the

135 other accessions (Extended Data Fig. 9). Nevertheless, the $\sim 13.1-\mathrm{kb}$ 5'UTR of Rps 11 was found

136 to match the putative 5'UTRs of two adjacent NLR genes in W05, suggesting that the large 5'UTR

137 of Rps11 was formed by fusion of the two 5'UTRs in PI 594527 after its divergence from W05

138 (Fig. 4, Box 3). In addition, the presence/absence of the conserved transcription start region (TSR)

139 is likely responsible for the drastic expression variations of the NLR genes in the Rps 11/ "rps 11"

140 region across the diverse soybean genomes (Fig. 4, Box 4, and Supplemental Table 5).

142 Discussion

143 We isolated Rps11 conferring an extremely broad spectrum of PRSR resistance in soybean using 144 a gap-free sequence-based fine mapping and cloning approach. Given such a magnitude of 145 structural and gene copy number variations of the NLR gene cluster and the lack of allelic copies 146 of the Rps 11 locus in the soybean pangenome, the sizable mapping population and the high quality 147 of genome assemblies of both parental lines have been essential for the success. As few NLR 148 clusters harboring known resistance loci have been fully sequenced and compared at the population 149 level, this study sheds light on the evolutionary plasticity and consequences of resistance genes 150 such as gain, loss, and reinforcement of resistance. Previously, two small NLR genes have been 151 reported to be responsible for Rps resistance conferred by the Rps $1-k$ locus in Williams $82^{15,16}$. 152 Intriguingly, neither of the two genes can be found in any versions/sources of the Williams 82 
153 genome assembly including unassembled contigs (soybase.org; 9). Thus, the candidate gene(s) of 154 Rpsl-k remains to be validated. In addition, Rpsl-k has become ineffective to many of newly 155 evolved $P$. sojae races (8). Therefore, the source of resistance conferred by Rps11 is highly 156 desirable. The LRR domains in NLR genes are involved in determining the plant's ability to 157 recognize specific pathogen effectors ${ }^{17,18}$, but whether the expanded LRR domain in Rps11 is 158 responsible for such a broad resistance spectrum remains to be tested. This gene appears to be a 159 suitable model for investigating molecular mechanisms underlying the extreme broadness of

160 resistance spectrum and for developing strategies such as such as engineering of NLR genes for 161 resistance enhancement. This study also provides Rps11-based unique markers for precise 162 selection of the gene and will accelerate the deployment of the gene for soybean protection

\section{Main references}

1651 Goheen, E. \& Frankel, S. Phytophthoras in forests and natural ecosystems. Gen. Tech. Rep. 166 PSW-GTR-221, 311-314 (2009).

1672 Nowicki, M., Foolad, M. R., Nowakowska, M. \& Kozik, E. U. Potato and tomato late blight 168 caused by Phytophthora infestans: an overview of pathology and resistance breeding. Plant 169 disease 96, 4-17 (2012).

1703 Dorrance, A. E. et al. Phytophthora root and stem rot of soybean. The Plant Health 171 Instructor, 1 (2007).

1724 Wrather, A. \& Koenning, S. Effects of diseases on soybean yields in the United States 1996 173 to 2007. Plant Health Progress 10, 24 (2009).

1745 Tyler, B. M. Phytophthora sojae: root rot pathogen of soybean and model oomycete.

175 Molecular plant pathology 8, 1-8 (2007). 
1766 Dorrance, A. \& Schmitthenner, A. New sources of resistance to Phytophthora sojae in the 177 soybean plant introductions. Plant Disease 84, 1303-1308 (2000).

1787 Abney, T. et al. New races of Phytophthora sojae with Rps 1-d virulence. Plant disease 81, $179 \quad 653-655$ (1997).

1808 Ping, J. et al. Identification and molecular mapping of Rps11, a novel gene conferring 181 resistance to Phytophthora sojae in soybean. Theoretical and Applied Genetics 129, 445-451 182 (2016).

1839 Chu, J. et al. Construction and analysis of eight soybean reference genomes reveal structural $184 \quad$ variations important for domestication and genetic breeding. Sientific Data (In press) (2021).

18510 Schmutz, J. et al. Genome sequence of the palaeopolyploid soybean. nature 463, 178-183 186 (2010).

18711 Xie, M. et al. A reference-grade wild soybean genome. Nature communications 10, 1-12 $188 \quad$ (2019).

18912 Shen, Y. et al. De novo assembly of a Chinese soybean genome. Science China Life Sciences $190 \quad 61,871-884(2018)$.

19113 Shen, Y. et al. Update soybean Zhonghuang 13 genome to a golden reference. Sci China Life 192 Sci 62, 1257-1260 (2019).

19314 Liu, Y. et al. Pan-genome of wild and cultivated soybeans. Cell 182, 162-176. e113 (2020).

19415 Gao, H. \& Bhattacharyya, M. K. The soybean-Phytophthora resistance locus Rps1-k 195 encompasses coiled coil-nucleotide binding-leucine rich repeat-like genes and repetitive 196 sequences. BMC Plant Biology 8, 1-14 (2008).

19716 Gao, H., Narayanan, N. N., Ellison, L. \& Bhattacharyya, M. K. Two classes of highly similar 198 coiled coil-nucleotide binding-leucine rich repeat genes isolated from the Rps1-k locus 
199 encode Phytophthora resistance in soybean. Molecular plant-microbe interactions 18, 1035-

$200 \quad 1045(2005)$.

20117 McHale, L., Tan, X., Koehl, P. \& Michelmore, R. W. Plant NBS-LRR proteins: adaptable 202 guards. Genome biology 7, 1-11 (2006).

20318 DeYoung, B. J. \& Innes, R. W. Plant NBS-LRR proteins in pathogen sensing and host

204 defense. Nature immunology 7, 1243-1249 (2006).

205

206 

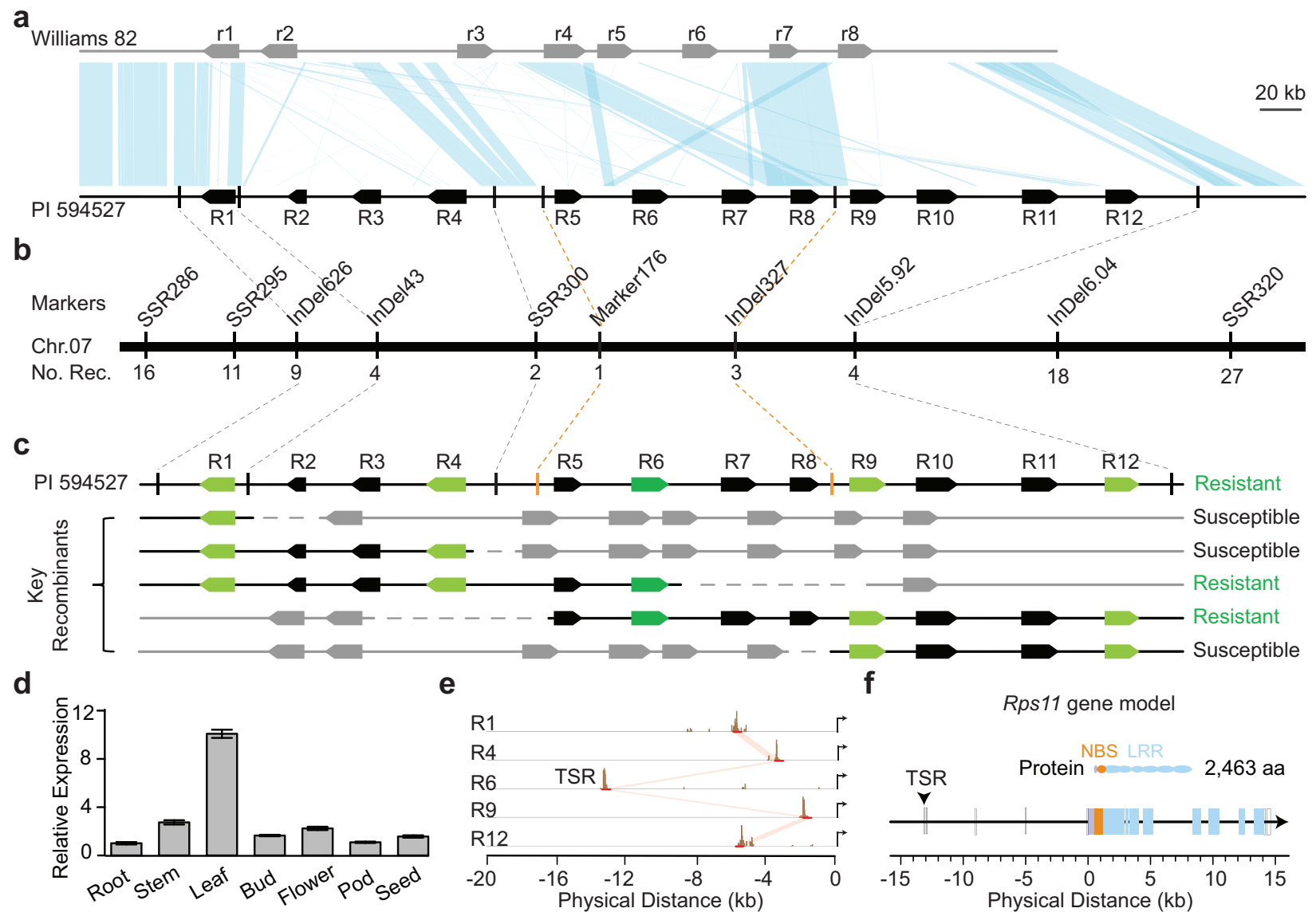

211 Fig. 1 | Map-based cloning of the Rps11 locus. a, Comparison of the NLR gene clusters between

212 Williams 82 and PI 594527. Black boxes represent NLR genes and light-blue shades represent

213 syntenic blocks between two genomes. b, Physical locations of the markers used for fine mapping

214 of the Rps11 locus. The black line represents the genome region in the Rps11 region on

215 chromosome 7. The name of each marker used for mapping were labeled above the line. Numbers

216 under the line indicate the number of recombinants at each marker. Dashed lines show the position

217 of the markers within the NLR gene cluster. The final mapping boundaries are highlighted by

218 orange dashed lines. c, Expression analyses in key recombinants. Green/light-green boxes 
219 represent the five expressed NLR gene from PI 594527, black boxes represent the non-expressed 220 NLR genes from PI 594527. Grey boxes represent the NLR genes from Williams 82. Dashed lines 221 represent unknow genotype. The phenotype of each key recombinant is indicated as resistant or 222 susceptible at the right side of each recombinant type. d, Expression profile of Rps 11 in different 223 tissues. The error bars represent standard errors. e, 5' Rapid amplification of cDNA ends (5'RACE) 224 performed for the 5 expressed NLR genes (R1, R4, R6, R9 and R12). $\mathrm{x}$ axis represents the distance 225 upstream of the first exon. Brown bars represents the 5' RACE reads mapped to each NLR gene. 226 Red lines/shades show the promoter regions sharing sequence similarity. Arrows at the right side 227 indicate the direction of the NLR genes. f, Gene model of Rps11 (R6). The vertical arrow points 228 to the transcription start region (TSR). Orange color indicates the region encoding the NBS 229 domain; Light-blue color indicates the regions encoding the LRR domains; Grey color indicates 230 the region without a domain detected. Open boxes represent UTRs. 

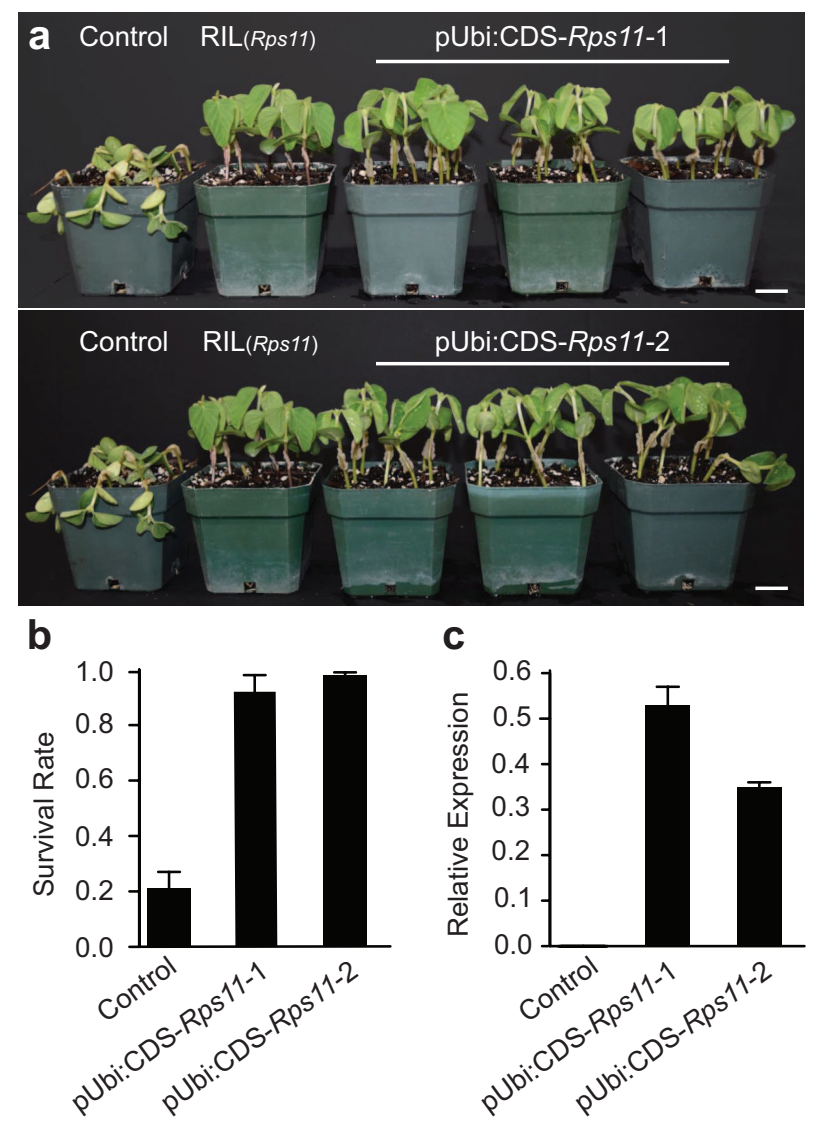

234 Fig. 2 | Complementation test results. a, Photographic illustration of the resistance in two 235 independent transgenic lines. In each transgenic event, three homozygous T2 families, a non236 transgenic line (Control), and a F5 RIL (Rps11/Rps11) were inoculated. Scale bars $=2.5 \mathrm{~cm}$. b, 237 Statistics of the resistance test of homozygous T2 families compared with non-transgenic lines 238 (Control). The $\mathrm{Y}$-axis is the survival rate after inoculation. The error bars represent standard errors. 239 c, Relative expression of the transgene (R6) compared to endo-reference gene in transgenic lines 240 and non-transgenic lines. The error bars represent standard errors. 


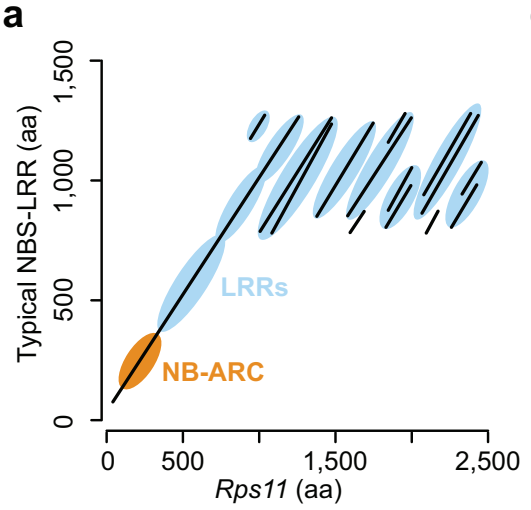

b

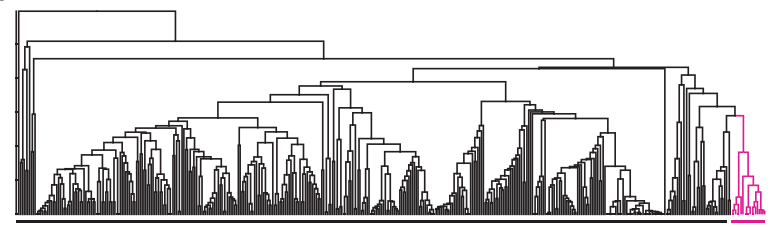

Typical NLR
C

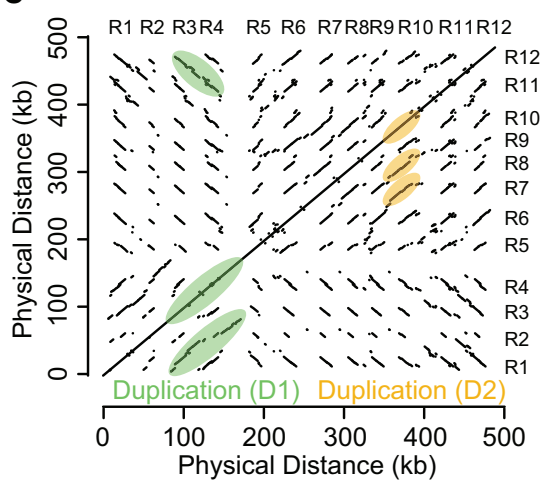

e d
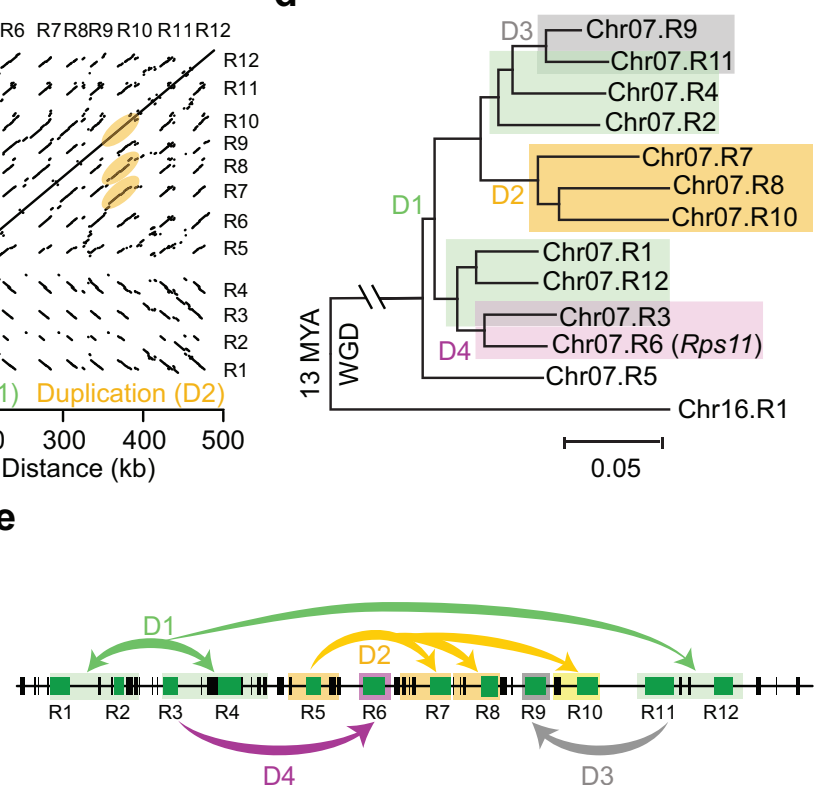

Fig. 3 | Evolutionary history of the giant NLR genes in the Rps11 locus. a, Comparison of

246 protein sequences between a giant NLR gene and a typical NLR gene. Lines represent the

247 alignments between two protein sequences. Orange color highlights the NB-ARC domain region.

248 Light-blue color highlights the LRR domain regions. b, Phylogenetic tree of all the NLR genes in

249 PI 594527 built using the conserved NB-ARC domain region. Giant NLR genes and typically sized

250 NLR genes are labeled. c, Dot plot of sequence comparison within the Rps11 region in PI 594527.

251 Light-green color highlights the segmental duplication of R1-R2, R3-R4 and R11-R12

252 (Duplication 1, D1). Light-orange color highlights the segmental duplication of R7, R8 and R10

253 (Duplication 2, D2). d, Phylogenetic relationship of all the NLR genes underlying Rps 11 and the

254 NLR gene, Chr16.R1, from its whole-genome duplication region, constructed using transcript

255 sequence. Background colors highlight the groups produced by each duplication event. e,

256 Illustration of the evolutionary history of the giant NLR gene cluster in the Rps 11 region. Green

257 boxes represent NLR genes and black boxes represent predicted non-NLR genes. 


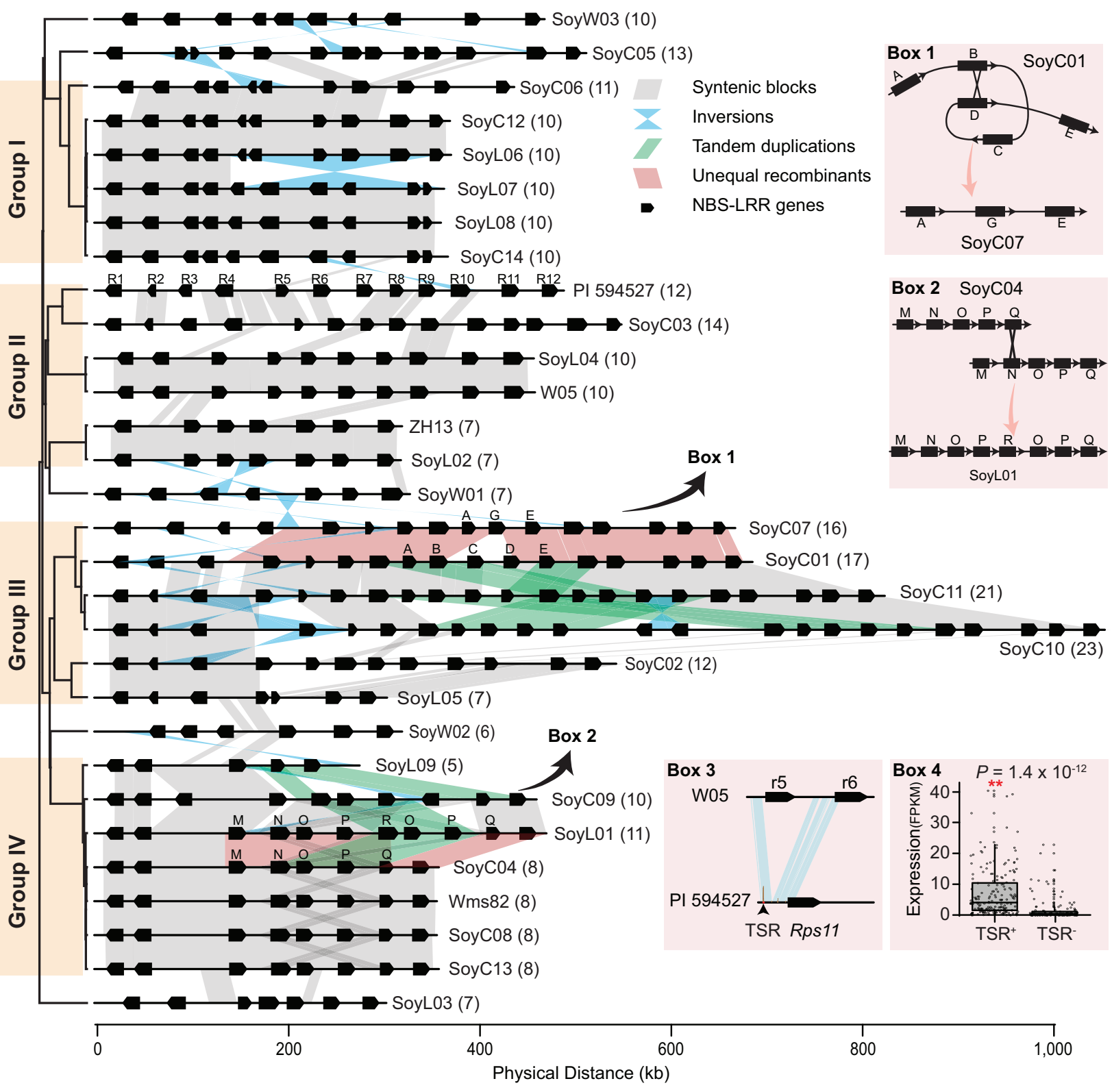

260 Fig. 4 | Diversification of the NLR gene cluster across 30 diverse soybean genomes. The

261 phylogenetic tree on the left side was built using SNPs data with the Rps11 region. Orange color

262 highlights the four major haplotype groups. Each black box represents an NLR gene. Grey shades

263 represent syntenic blocks among genomes. Light-blue highlight inversion events. Green highlight

264 tandem duplication events. Light-red highlight potential unequal recombination events. Box 1 and

265 Box 2 exemplify a deletion and a duplication event induced by unequal recombination, 
266 respectively. The name of each genome was labeled at right side of each cluster and the numbers

267 in parentheses are the total copy number of NLR genes in each genome at the Rps 11 corresponding 268 region. Box 3 shows the sequence comparison of the promoter regions of Rps 11 and that of W05.r5

269 and W05.r6. Light-blue shades represent syntenic blocks. Box 4 shows the comparison of the 270 expression levels of NLR genes with TSR (TSR ${ }^{+}$) and without TSR (TSR) in the Rps11/ "rps 1 1"

271 region across all the genomes. Red asterisks indicate the significance and $P$ value is shown above 272 the plot.

273

\section{Methods}

\section{Plant materials and resistance evaluation}

276 The mapping populations were generated from an initial cross between PI 594527 and Williams.

277 In total, 17,050 $\mathrm{F}_{3}$ plants derived from heterozygous $\mathrm{F} 2$ individuals were screened for

278 identification of recombinants. Only the recombinants with one side heterozygous and the other

279 side homozygous Williams genotype were used for fine mapping, because the two expected

280 phenotypes could be accurately distinguished. About 30 seedlings from each line were

281 inoculated with Race 1 of $P$. sojae using a protocol previously described ${ }^{3,19}$. Lines with less than

$28225 \%$ of progenies surviving after inoculation were classified as susceptible; recombinants with

$28325-75 \%$ of progenies surviving were classified as segregating, lines with more than $75 \%$ of

284 progenies surviving after inoculation were classified as completely resistant.

286 Genotyping the recombinants.

287 SSR markers and insertion/deletion markers (InDel) were identified and designed based on re-

288 sequencing data of the two parental lines. Marker_176kb was a dominant marker that could only 
289 be amplified from the donor line. Only the markers with a unique hit at the Rps 11 region were

290 used for fine mapping. Kompetitive allele specific PCR (KASP) makers were also used to

291 identify and genotype the recombinants. All makers used in this study are listed in Supplemental

292 Table 6.

293

\section{Long and short read genome sequencing}

295 Long-read data was generated using the Pacific BioSciences (Menlo Park, CA, USA) Sequel

296 platform at Corteva Agriscience ${ }^{\mathrm{TM}}$ (Johnston, IA, USA). Eight SMRT cells were performed with

297 10-hr movies and v6 chemistry. Raw subreads were filtered to a minimum of $12 \mathrm{~kb}$ generating

$29877 \times$ genome coverage. The raw subread N50 length was $28.9 \mathrm{~kb}$. Linked short-read data were

299 generated by sequencing 10X Genomics (Pleasanton, California) Chromium libraries at Corteva

300 Agriscience ${ }^{\mathrm{TM}}$ on the Illumina (San Diego, California) HiSeq2500 platform in a PE151

301 configuration. The coverage depth and mean molecule length for the Chromium library were

$30245.2 \times$ and $93.8 \mathrm{~kb}$, respectively.

\section{Genome assembly and sequence polishing}

305 Canu ${ }^{20} \mathrm{v} 1.8$ (https://github.com/marbl/canu) was used to self-correct the raw subreads and to

306 assemble the corrected reads into contigs. The following changes were made to the default

307 parameters: correctedErrorRate $=0.065$, corMhapSensitivity $=$ normal, and ovlMerDistinct $=0.99$.

308 A minimum contig length of $30 \mathrm{~kb}$ was applied. Additional sequence polishing was performed

309 by aligning raw PacBio subreads to the contig assembly using pbmm2 v0.12.0

310 (https://github.com/PacificBiosciences/pbmm2) and applying the Arrow algorithm from the

311 Genomic Consensus package v2.3.2 (https://github.com/PacificBiosciences/GenomicConsensus) 
312 to identify and correct remaining consensus errors in the contigs. These tools were acquired from

313 pbbioconda (https://github.com/PacificBiosciences/pbbioconda). The consensus sequence

314 accuracy was further enhanced by complementing the long read contig assembly with Chromium

315 linked short-reads. Chromium datasets were aligned to contigs using Long Ranger v2.2.2. The

316 sequence assembly polishing tool Pilon ${ }^{21}$ v1.22 (https://github.com/broadinstitute/pilon) was

317 used to correct individual base errors and small indels from the Chromium data aligned to the 318 contigs using the "--fix bases -minmq 30" parameters.

\section{Creating genome maps}

321 Genome maps were generated in the Bionano Saphyr platform at Corteva Agriscience ${ }^{\mathrm{TM}}$ using

322 the Direct Label and Stain (DLS) approach ${ }^{22}$. Nuclear DNA was isolated from leaf tissue using a 323 modified version of the Bionano Prep ${ }^{\mathrm{TM}}$ Plant Tissue DNA Isolation protocol

324 (https://Bionanogenomics.com/wp-content/uploads/2017/01/30068-Bionano-Prep-Plant-Tissue-

325 DNA-Isolation-Protocol.pdf) that did not include a gradient centrifugation step. DLE-1-labeled

326 molecule data were filtered to create a dataset with a molecule N50 of $441 \mathrm{~kb}$ and 267×

327 coverage. The resulting genome maps were filtered to remove coverage and length outliers. The

328 final genome map assembly consisted of 43 maps with a genome map N50 of $26.7 \mathrm{Mb}$ and a

329 total map length of $985 \mathrm{Mb}$. This dataset was assembled via the Bionano Genomics Access

330 software platform (Solve3.2.2_08222018) with the configuration file

331 optArguments_nonhaplotype_noES_noCut_DLE1_saphyr.xml.

332

333 Hybrid scaffolding of genome maps with sequence contigs. 
334 Hybrid scaffolds were generated from the polished contigs and the Bionano genome maps using

335 the Bionano Genomics Access software (Solve3.3_10252018) and the DLE-1 configuration file

336 hybridScaffold_DLE1_config.xml. In addition to auto-conflict resolution performed by the

337 software, manual curation was performed to resolve overlapping and embedded contigs by

338 providing additional "Conflict resolutions" and re-running the hybrid scaffolding. In the final

339 product, the assembly had 43 hybrid scaffolds (Scaffold N50=26.4 Mb, Total scaffold length=

$340978.1 \mathrm{Mb}$ ) with 229 leftover contigs that were not scaffolded with a combined length of 21.3

$341 \mathrm{Mb}$.

342

343 Building chromosome-scale pseudomolecules

344 A reference-based approach was feasible to create chromosome-scale pseudomolecules using the

345 Glycine max Wm82.a2.v1 reference assembly (https://phytozome.jgi.doe.gov/), because only an

346 average of 2.15 scaffolds per chromosome needed to be placed. To map hybrid scaffolds to the

347 reference, each scaffold was chunked into 100-bp fragments and then aligned to the reference

348 genome using minimap2 ${ }^{23} \mathrm{v} 2.10$ (https://github.com/lh3/minimap2). Then, a custom script was

349 used to determine the chromosome position and orientation for each scaffold based on the

350 alignment of each "chunked scaffold cloud". All scaffolds were able to be placed using this

351 method. Leftover unscaffolded contigs were concatenated with 100-bp N-gaps and assigned to

352 Chr00.

353

\section{$354 \quad$ NLR gene annotation and expression analysis}

355 NLR genes were annotated using NLR-Annotator ${ }^{24}$. RNA samples were extracted from mixed

356 stem tissues from seedlings of each key recombinants using RNeasy Plant Mini Kit (Cat No. 
74904, Qiagen) and were treated with RNase-Free DNase Set (Cat No. 79254, Qiagen) to

358 remove DNA. RNA-seq was performed by Purdue Genomic Core Facility

359 (https://www.purdue.edu/hla/sites/genomics/). RNA-seq data were mapped to the genome of the

360 donor line using STAR $^{25}$ and expression was calculated based on the number of reads mapped to

361 each NLR gene.

362

363 Plasmid Construction and Transformation

364 To make the over-expression construct for the Rps11 candidate gene R6, the CDS of R6 was

365 synthesized by Genscript as 3 fragments and assembled with AtUbi3 promoter and Gateway

366 ATT sites by homologous recombination in yeast to make a Gateway entry vector ${ }^{26}$. This was

367 recombined into a Gateway destination vector by LR reaction using Gateway Technology with

368 Clonase II (25-0749, Invitrogen) for transformation into Ochrobactrum. Ochrobactrum-mediated

369 soybean embryonic axis transformation was done as previously described (US20180216123A1;

370 WO2020/005933A1; WO2020/092494A1). Mature dry seeds of soybean cultivar 93 Y21 were

371 disinfected using chlorine gas and imbibed on semi-solid medium containing $5 \mathrm{~g} / 1$ sucrose and 6

$372 \mathrm{~g} / \mathrm{l}$ agar at room temperature in the dark. After an overnight incubation, the seed was soaked in

373 distilled water for an additional 3-4 hrs at room temperature in the dark. Intact embryonic axis

374 explants were isolated and transferred to the deep plate with $15 \mathrm{~mL}$ of Ochrobactrum

375 haywardense H1-8 suspension (OD 0.5 at $600 \mathrm{~nm}$ ) in infection medium composed of 1/10X

376 Gamborg B5 basal medium, $30 \mathrm{~g} / \mathrm{L}$ sucrose, $20 \mathrm{mM}$ MES, $0.25 \mathrm{mg} / \mathrm{L}$ GA3, $1.67 \mathrm{mg} / \mathrm{L}$ BAP, 200

$377 \mu \mathrm{M}$ acetosyringone and $1 \mathrm{mM}$ dithiothreitol in $\mathrm{pH}$ 5.4. The plates were sealed with parafilm

378 ("Parafilm M" VWR Cat\#52858), then sonicated (Sonicator-VWR model 50T) for 30 seconds.

379 After sonication, embryonic axis explants were transferred to a single layer of autoclaved sterile 
filter paper (VWR\#415/Catalog \# 28320-020). The plates were sealed with Micropore tape

381 (Catalog \# 1530-0, 3M, St. Paul, MN)) and incubated under dim light (5-10 $\mu \mathrm{E} / \mathrm{m} 2 / \mathrm{s}$, cool white

382 fluorescent lamps) for $16 \mathrm{hrs}$ at $21^{\circ} \mathrm{C}$ for 3 days.

383

384 After co-cultivation, the base of each embryonic axis was embedded in shoot induction medium

385 (R7100, PhytoTech Labs) containing $30 \mathrm{~g} / \mathrm{L}$ sucrose, $6 \mathrm{~g} / \mathrm{L}$ agar and $25 \mathrm{mg} / \mathrm{L}$ spectinomycin

386 (S742, PhytoTech Labs) as a selectable agent and $500 \mathrm{mg} / \mathrm{L}$ cefotaxime (GoldBio, ST Louis,

$387 \mathrm{MO}, \mathrm{USA}$ ) in $\mathrm{pH}$ 5.7. Shoot induction was carried out at $26^{\circ} \mathrm{C}$ with a photoperiod of $18 \mathrm{hrs}$ and a

388 light intensity of 40-70 $\mu \mathrm{E} / \mathrm{m} 2 / \mathrm{s}$. After $4-6$ weeks in selection medium, the spectinomycin-

389 resistant shoots were cut and transferred to $1 / 2$ strength MS rooting medium (M404, PhytoTech

390 Labs) containing $15 \mathrm{~g} / \mathrm{L}$ sucrose, agar $6 \mathrm{~g} / \mathrm{L}, 10 \mathrm{mg} / \mathrm{L}$ spectinomycin and $250 \mathrm{mg} / \mathrm{L}$ cefotaxime

391 for further shoot and root elongations.

392

393 Marker-free transgenic soybean plants were generated by the Cre-lox site-specific recombination

394 system using heat shock treatment. For heat shock treatment of soybean, 2-4 cm T0 plantlets

395 with roots in $100 \times 25 \mathrm{~mm}$ Petri dishes on spectinomycin free-rooting medium were transferred

396 into a Percival incubator (Percival Scientific, Perry, IA, USA) at $45^{\circ} \mathrm{C}, 70 \%$ humidity for 2 hrs

397 in the dark. After the heat shock treatment, T0 plantlets were transferred to moistened Berger

398 BM2 soil (Berger, Saint-Modeste, QC, Canada), and kept enclosed in clear plastic tray boxes in a

399 Percival incubator at $26^{\circ} \mathrm{C}$ with a 16 -hr photoperiod at $250-350 \mu \mathrm{E} / \mathrm{m} 2 / \mathrm{s}$. Leaf punch samples

400 were collected for molecular analyses from newer growth 2 weeks after acclimatization of T0

401 events. Hardened plantlets were potted in 2-gallon pots containing moistened SunGro 702 and

402 grown to maturity for harvest in a greenhouse. The presence of the construct in the transgenic 
403 plants was confirmed by PCR with primers specific to the cloning vector and expression analysis

404 of R6 in the transgenic plants by qPCR.

405

406 Data and materials availability

407 All the raw sequence data and the genome assembly from this article have been deposited in NCBI 408 database under BioProject PRJNA718574. Novel biological materials described in this publication 409 may be available to the academic community and other not-for-profit institutions solely for non410 commercial research purposes upon acceptance and signing of a material transfer agreement 411 between the author's institution and the requestor. In some cases, such materials may originally 412 contain genetic elements described in the manuscript that were obtained from a third party(s), and 413 the authors may not be able to provide materials including third party genetic elements to the 414 requestor because of certain third-party contractual restrictions placed on the author's institution. 415 In such cases, the requester will be required to obtain such materials directly from the third party. 416 The authors and authors' institution do not make any express or implied permission(s) to the 417 requester to make, use, sell, offer for sale, or import third party proprietary materials. Obtaining 418 any such permission(s) will be the sole responsibility of the requestor. In order to protect Corteva 419 Agriscience $^{\mathrm{TM}}$ proprietary germplasm, such germplasm will not be made available except at the 420 discretion of Corteva Agriscience ${ }^{\mathrm{TM}}$ and then only in accordance with all applicable governmental 421 regulations. Plant germplasm and transgenic material will not be made available except at the 422 discretion of the owner and then only in accordance with all applicable governmental regulations. 423

\section{Methods references}


42519 Lin, F. et al. Molecular mapping of two genes conferring resistance to Phytophthora sojae

426 in a soybean landrace PI 567139B. Theoretical and applied genetics 126, 2177-2185

$427 \quad(2013)$.

42820 Koren, S. et al. Canu: scalable and accurate long-read assembly via adaptive k-mer

$429 \quad$ weighting and repeat separation. Genome research 27, $722-736$ (2017).

43021 Walker, B. J. et al. Pilon: an integrated tool for comprehensive microbial variant

431 detection and genome assembly improvement. PloS one 9, e112963 (2014).

43222 Ou, S. et al. Effect of sequence depth and length in long-read assembly of the maize

433 inbred NC358. Nature communications 11, 1-10 (2020).

43423 Li, H. Minimap2: pairwise alignment for nucleotide sequences. Bioinformatics 34, 3094$435 \quad 3100(2018)$.

43624 Steuernagel, B. et al. The NLR-Annotator tool enables annotation of the intracellular 437 immune receptor repertoire. Plant physiology 183, 468-482 (2020).

43825 Dobin, A. et al. STAR: ultrafast universal RNA-seq aligner. Bioinformatics 29, 15-21 $439 \quad(2013)$.

44026 Muller, H. et al. in Gene Synthesis 133-150 (Springer, 2012).

442 Acknowledgments

443 This work was mainly supported by Corteva Agriscience ${ }^{\mathrm{TM}}$. We would like to thank Jon Massman 444 and John Woodward for their leadership in the Purdue University-Corteva Agriscience project 445 collaboration. We thank Ajit Nott, Lyudmila Sidorenko, Abhijit Sanyal, Jon Allen and Tyler 446 Engelhart for their technical support in transformation, sequence analysis, resistance screening and 
447 greenhouse coordination. We also thank Dr. Xianzhong Feng for providing the Williams 82 448 assembly v3.0.

449

450 Author contributions

$451 \mathrm{JM}, \mathrm{RA}, \mathrm{OC}$, Jesse M, AB and JT designed the research. WW, LC, KF, Jon M, JB, XW, VL, CC, $452 \mathrm{TF}, \mathrm{DO}, \mathrm{AH}, \mathrm{DD}$, and GC performed the experiments. WW, LC, KF, XW analyzed the data. JM 453 and WW wrote the manuscript.

454

455 Competing interests: Patent protection has been and/or may be sought for work disclosed in this 456 manuscript.

457

458 Additional information

459 Supplemental Information is available for this paper.

460

461 Correspondence and requests for materials should be addressed to J.M. (maj@purdue.edu) or

462 R.A. (rajat.aggarwal@,corteva.com) 


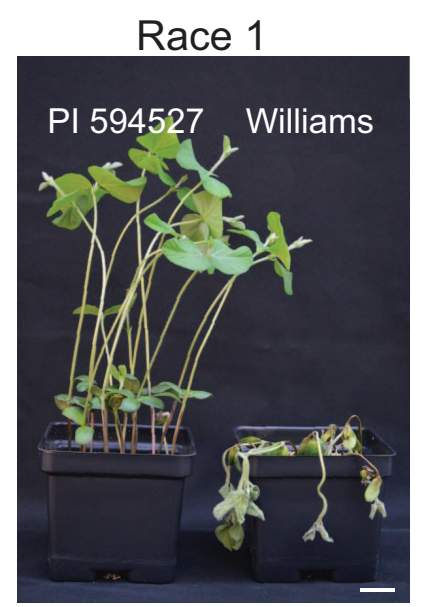

Race 25

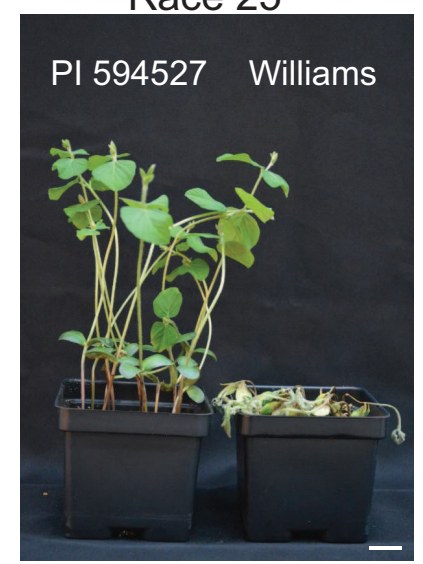

Race 31

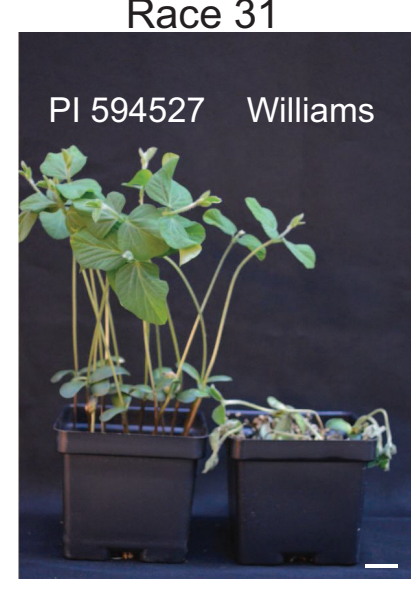

Race 3

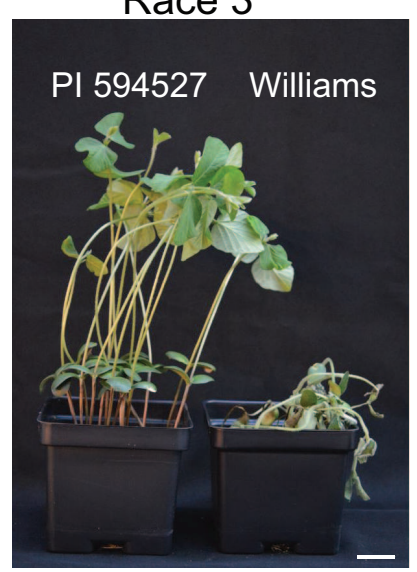

Race 17

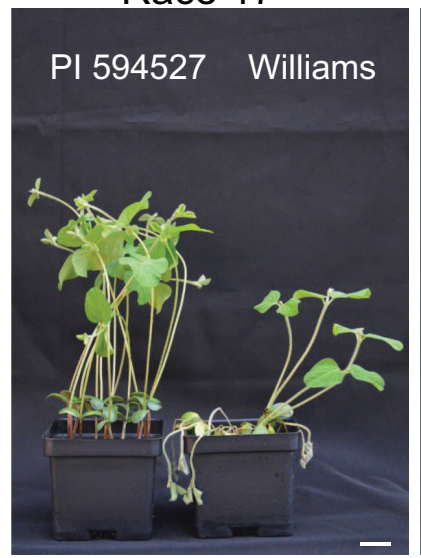

MINI 2002.01.05
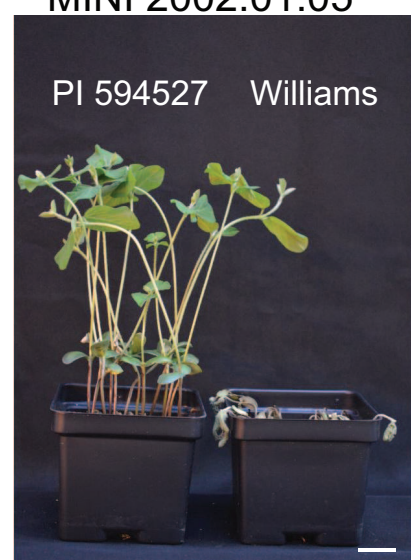

Race 4

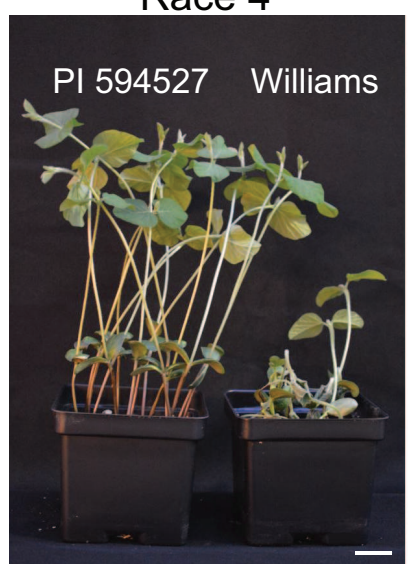

ISA 17D-1

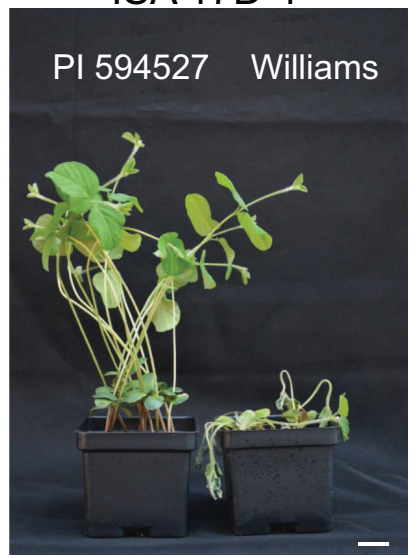

MINI 2003.08.01

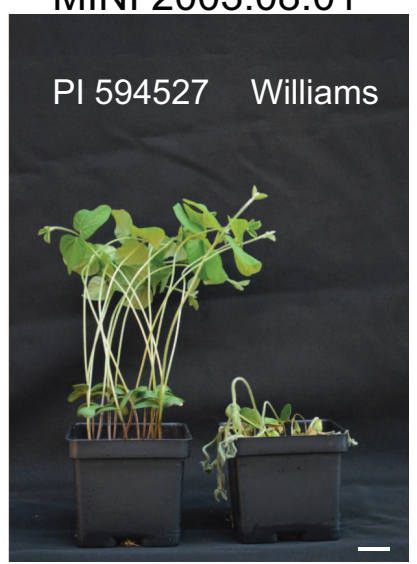

Race 7

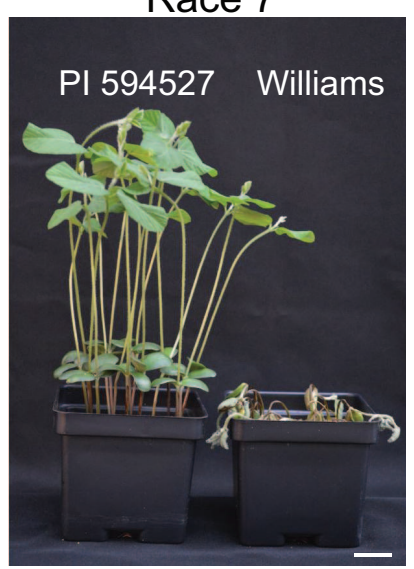

ISA 19A-1

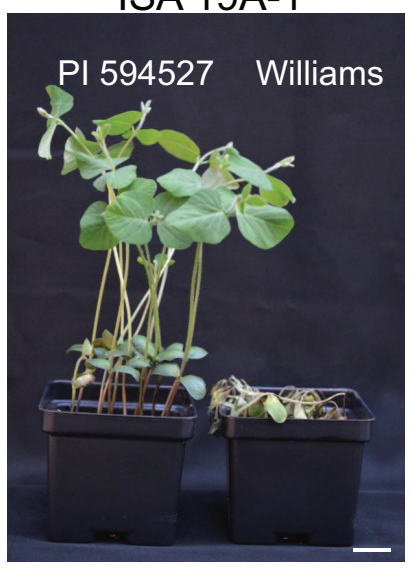

MINI 2004.03.01

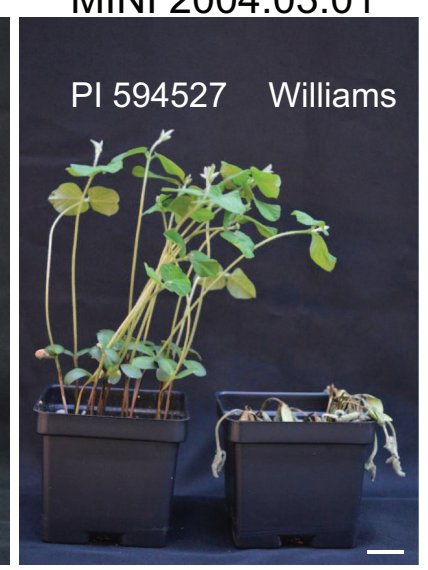

464 Extended Data Fig. 1 | Resistance spectrum of the Rps11 donor line. The Rps11 donor line,

465 PI594527 (left), and the susceptible control line, Williams (right), were inoculated with 12

466 races/isolates of $P$. sojae. Photos were taken 7 days after inoculation. Scale bars $=2.5 \mathrm{~cm}$. 


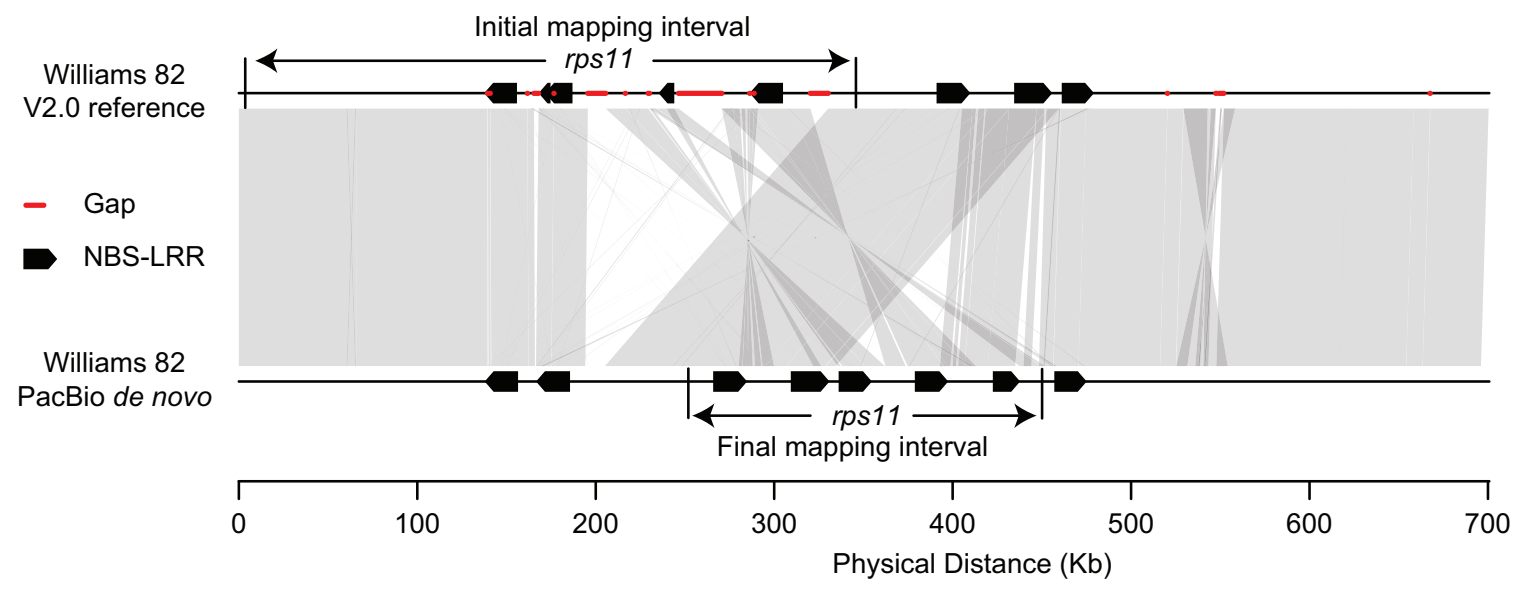

468 Extended Data Fig. 2 | Sequence comparison between Williams 82 assembly v2.0 and

469 assembly v3.0. Black boxes represent NBS-LRR genes and grey shades represent syntenic

470 blocks between two versions. Red lines/dots represent sequencing gaps in the assembly v2.0. 

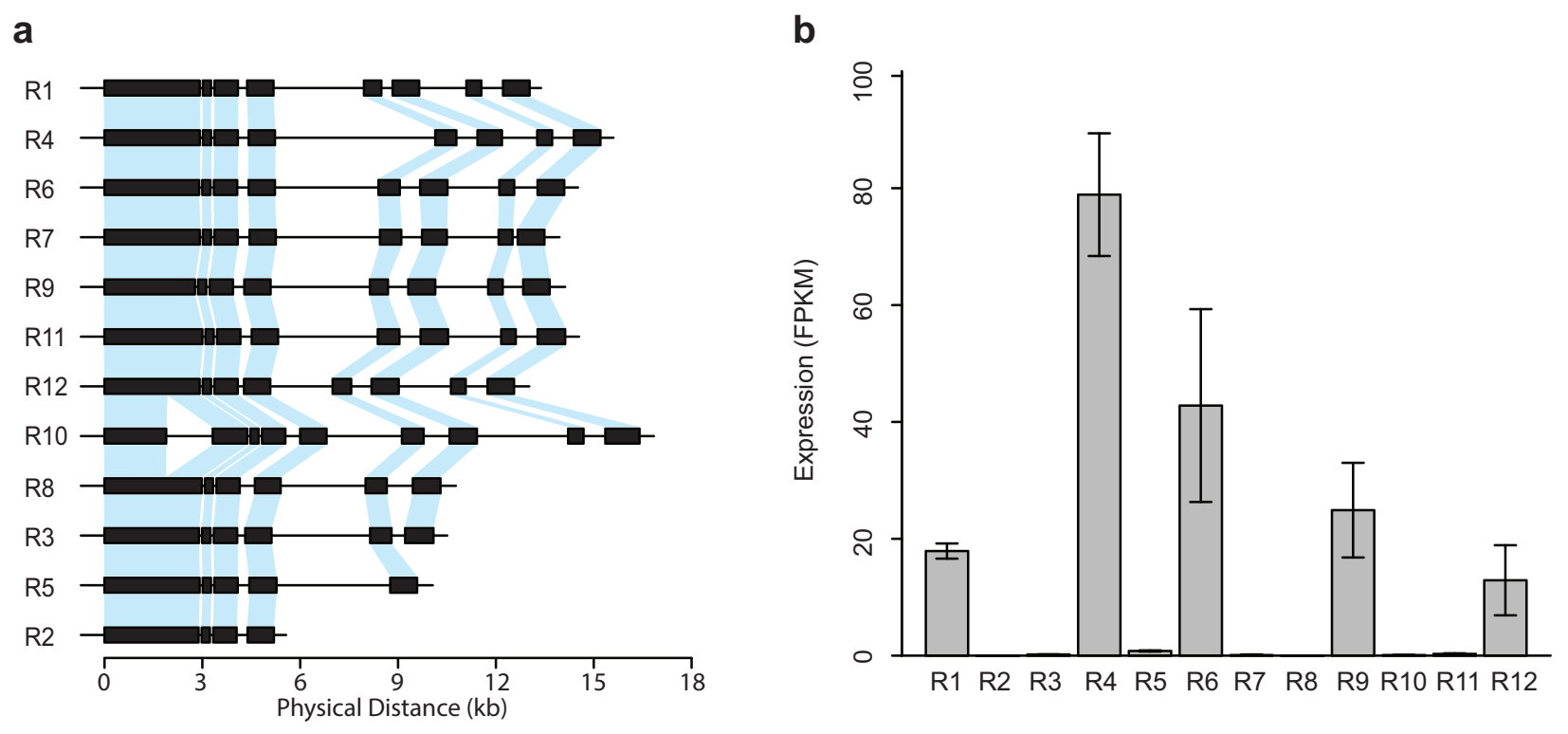

473 Extended Data Fig. 3 | Gene models and expression pattern of the NBS-LRR genes in the

474 Rps11 region. a, Gene models and alignments among twelve NBS-LRR genes in PI 594527.

475 Black boxes represent exons of each NBS-LRR gene. Light-blue shades represent alignments. b,

476 Expression profile of the twelve NBS-LRR genes in the Rps11 region. $y$-axis is the expression

477 level (FPKM) based on RNA-seq data. The error bars represent standard errors. 


\section{Williams82}

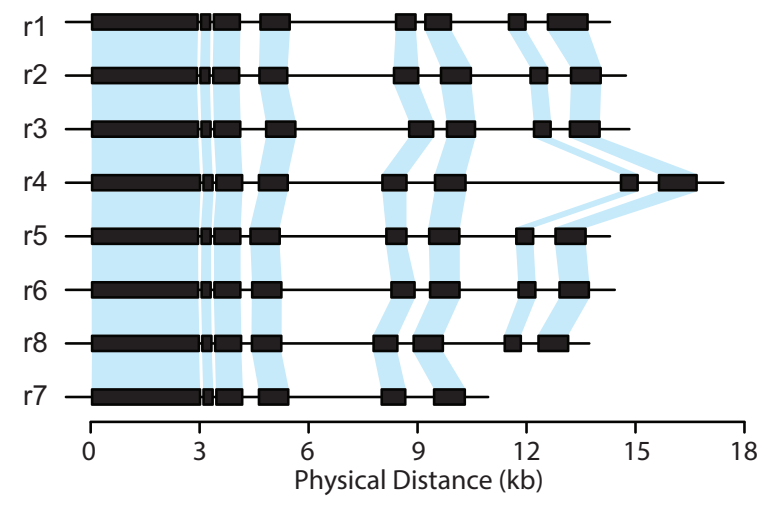

481 Extended Data Fig. 4 | Gene models of the eight NBS-LRR genes in the Rps11

482 corresponding region in Williams 82. Black boxes represent exons of each NBS-LRR gene.

483 Light-blue shades represent alignments. 
a
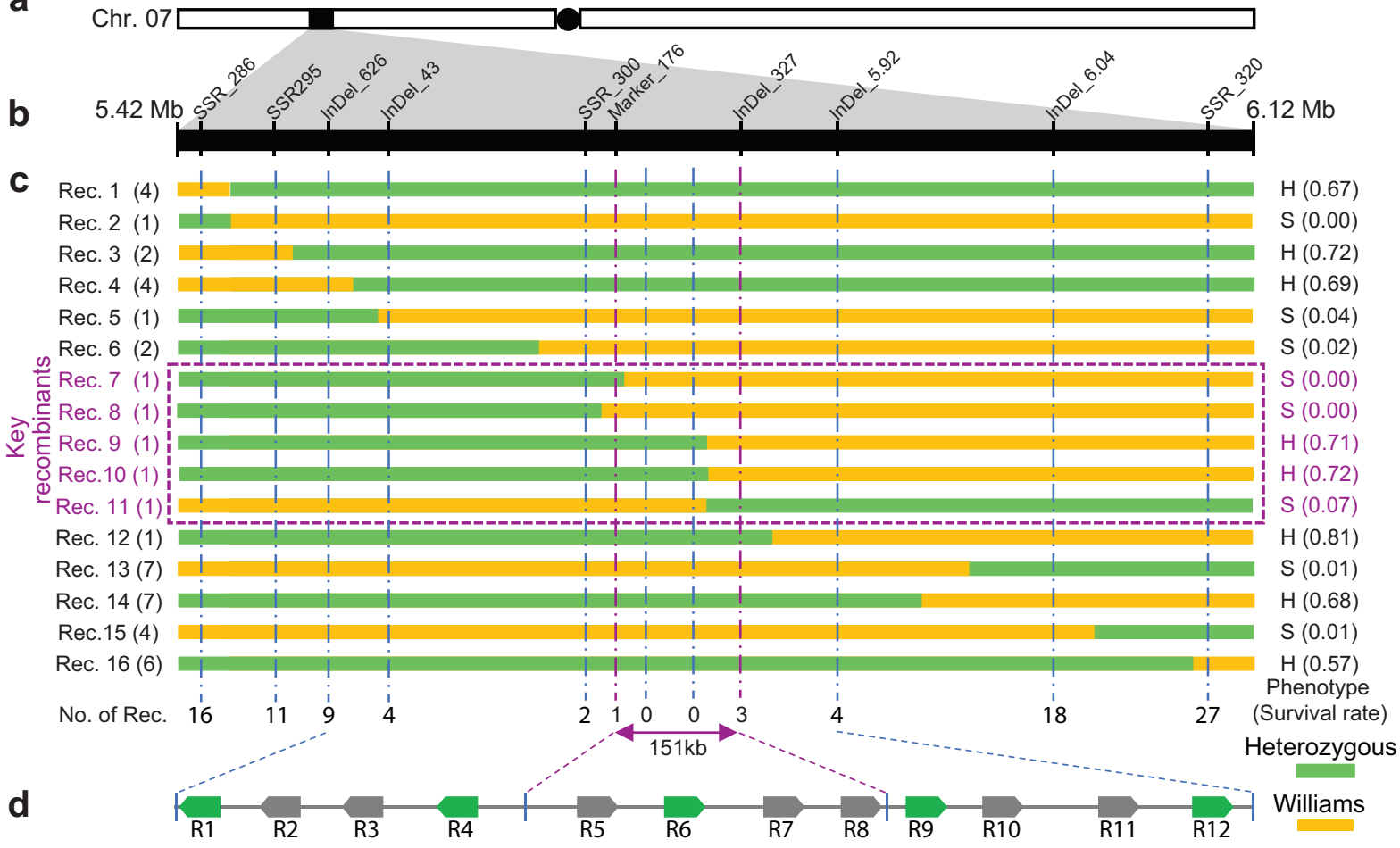

487 Extended Data Fig. 5 | Fine mapping of the Rps11 locus. a, Physical position of the Rps11

488 locus on chromosome 7. b, The markers used for fine mapping. c, Genotype and phenotype of

489 the recombinants. The numbers in parentheses at left side are the number of recombinants with

490 same haplotype. Green bars represent heterozygous genotype. Orange bars represent Williams

491 genotype. Phenotype (survival rate after inoculation) of each recombinant type are shown at right

492 side. H means heterozygous, S means susceptible. Numbers under each dashed line are the

493 number of recombinants at each marker. d, NBS-LRR gene cluster in the Rps 11 region. Green

494 bars represent expressed NBS-LRR genes. Grey bars represent non-expressed NBS-LRR genes. 


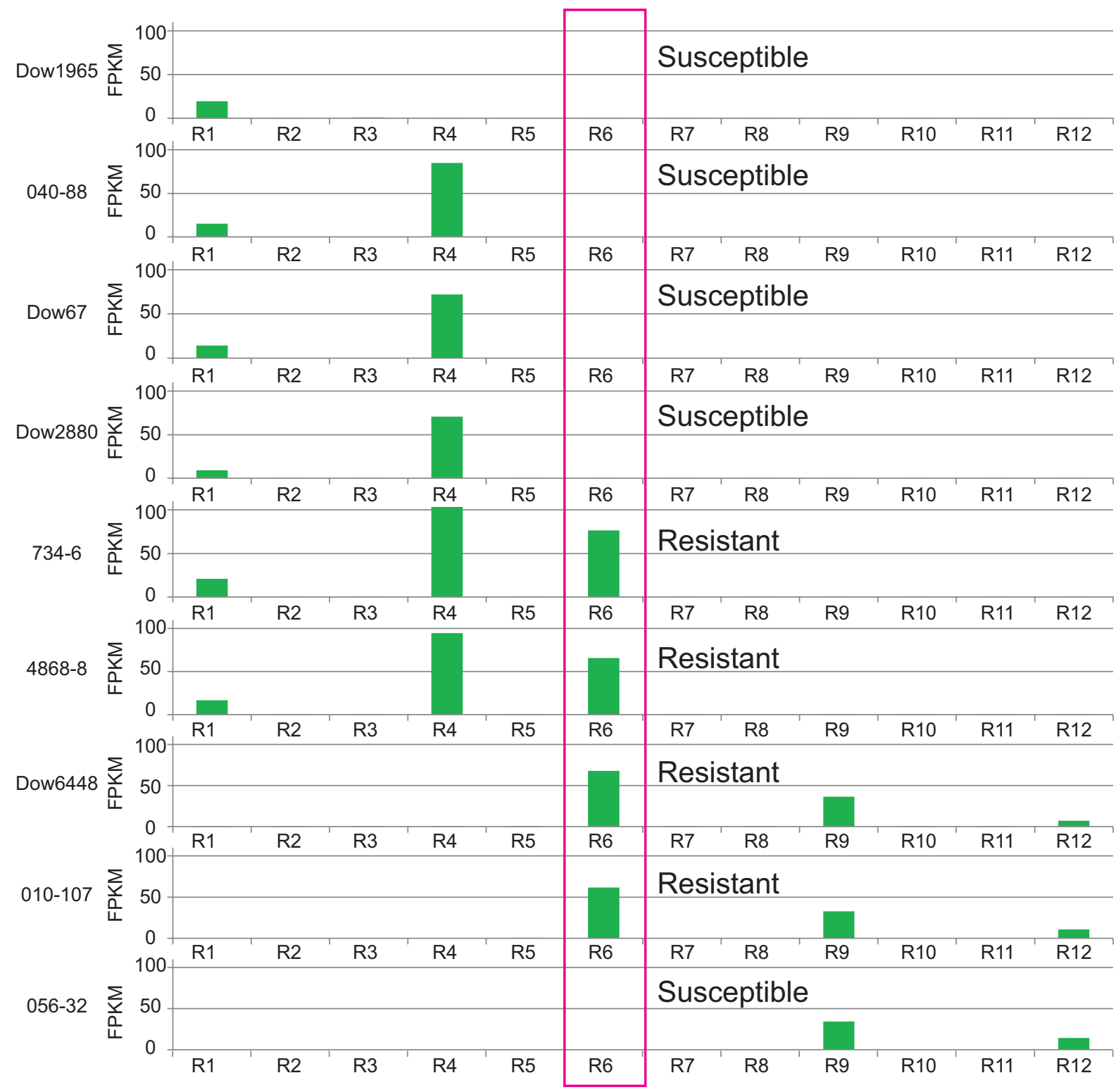

497 Extended Data Fig. 6 | Detection of the expression of the twelve NBS-LRR genes in each

498 key recombinant. The phenotype of each recombinant is labeled. $y$-axis is expression level

499 (FPKM) based on RNA-seq data. 


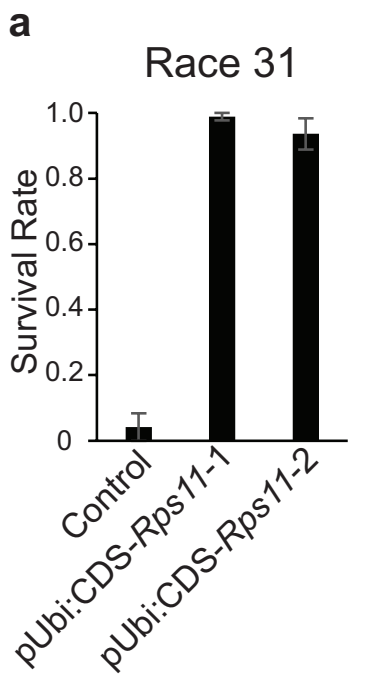

b

Race $06-03$

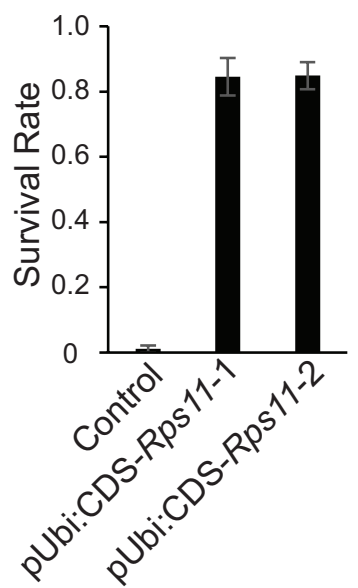

501 Extended Data Fig. 7 | Inoculation results of the transgenic lines with race 31 and Race 06-03.

502 a, Resistance to Race 31 . b, Resistance to Race 06-03. $y$-axis is the survival rate after

503 inoculation. The error bars represent standard errors. 

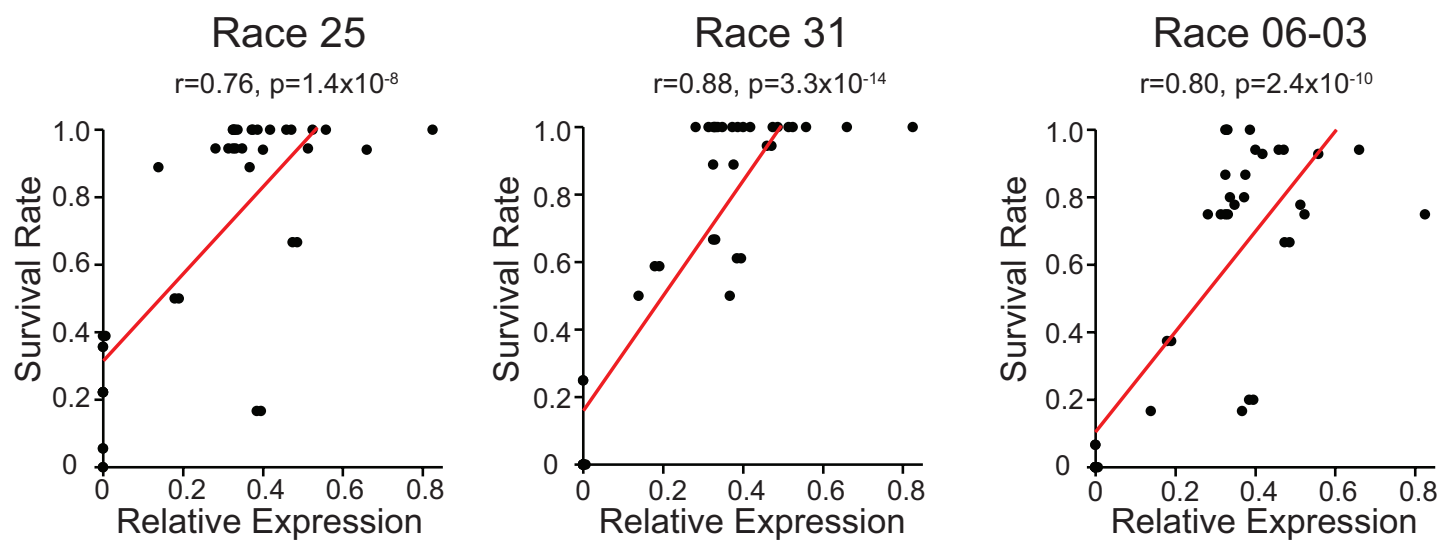

505 Extended Data Fig. 8 | Correlation between the expression of R6 (Rps 11) and the survival rate

506 after inoculation in T2 population. $x$-axes are relative expression of R6, and $y$-axes are survival

507 rate after inoculation with Race 25 (left), Race 31 (middle), Race 06-03 (right). 

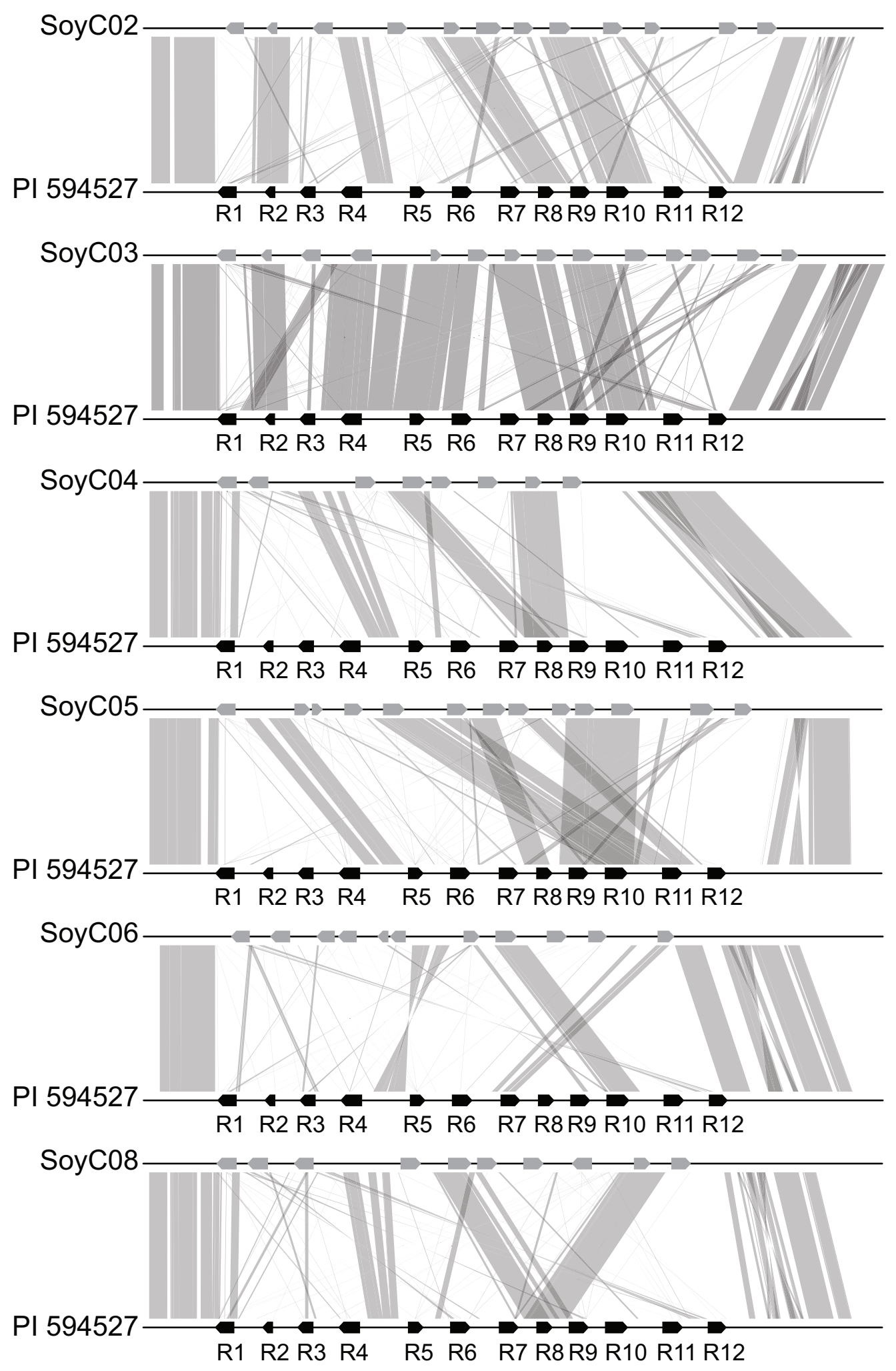

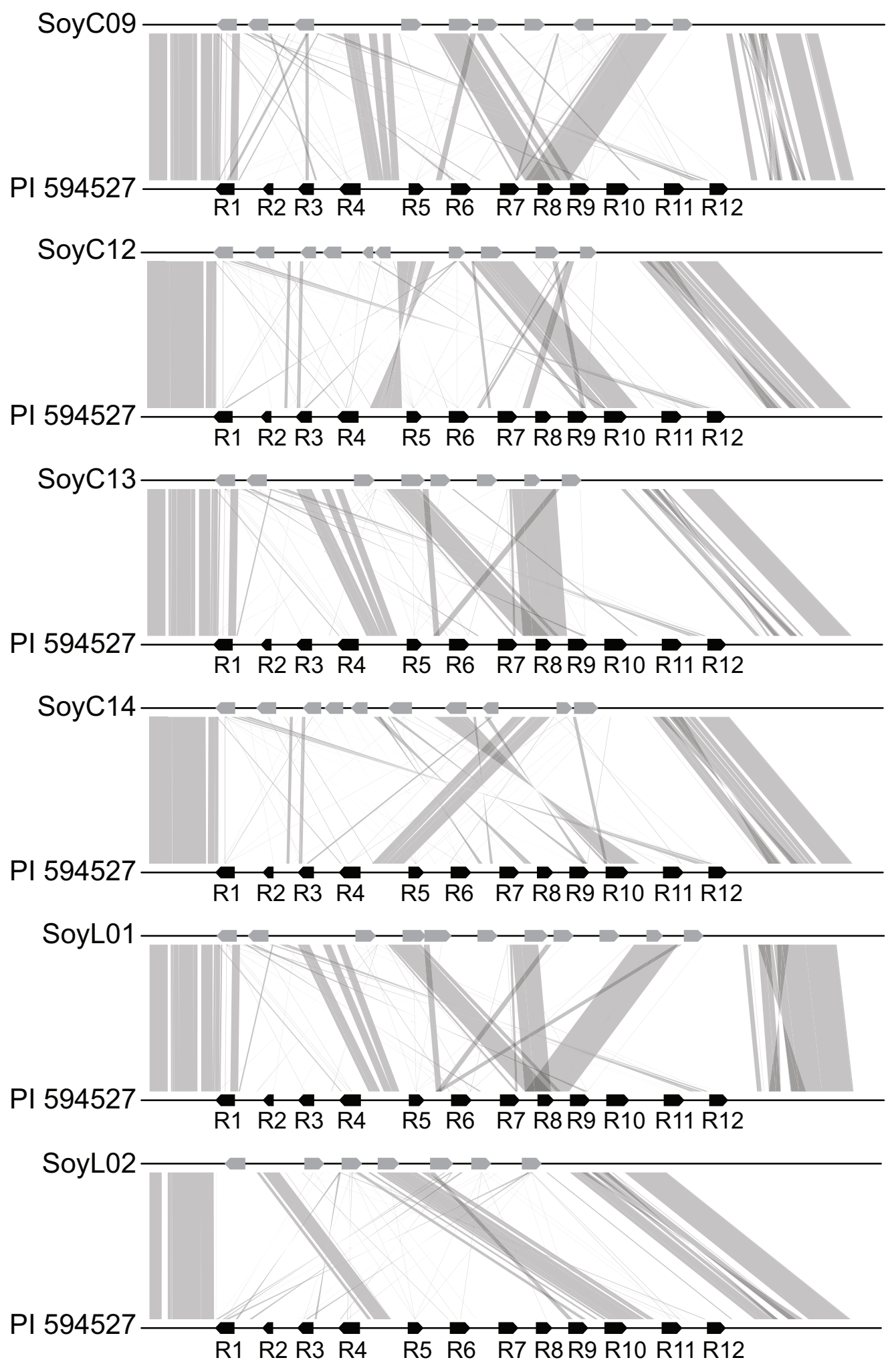

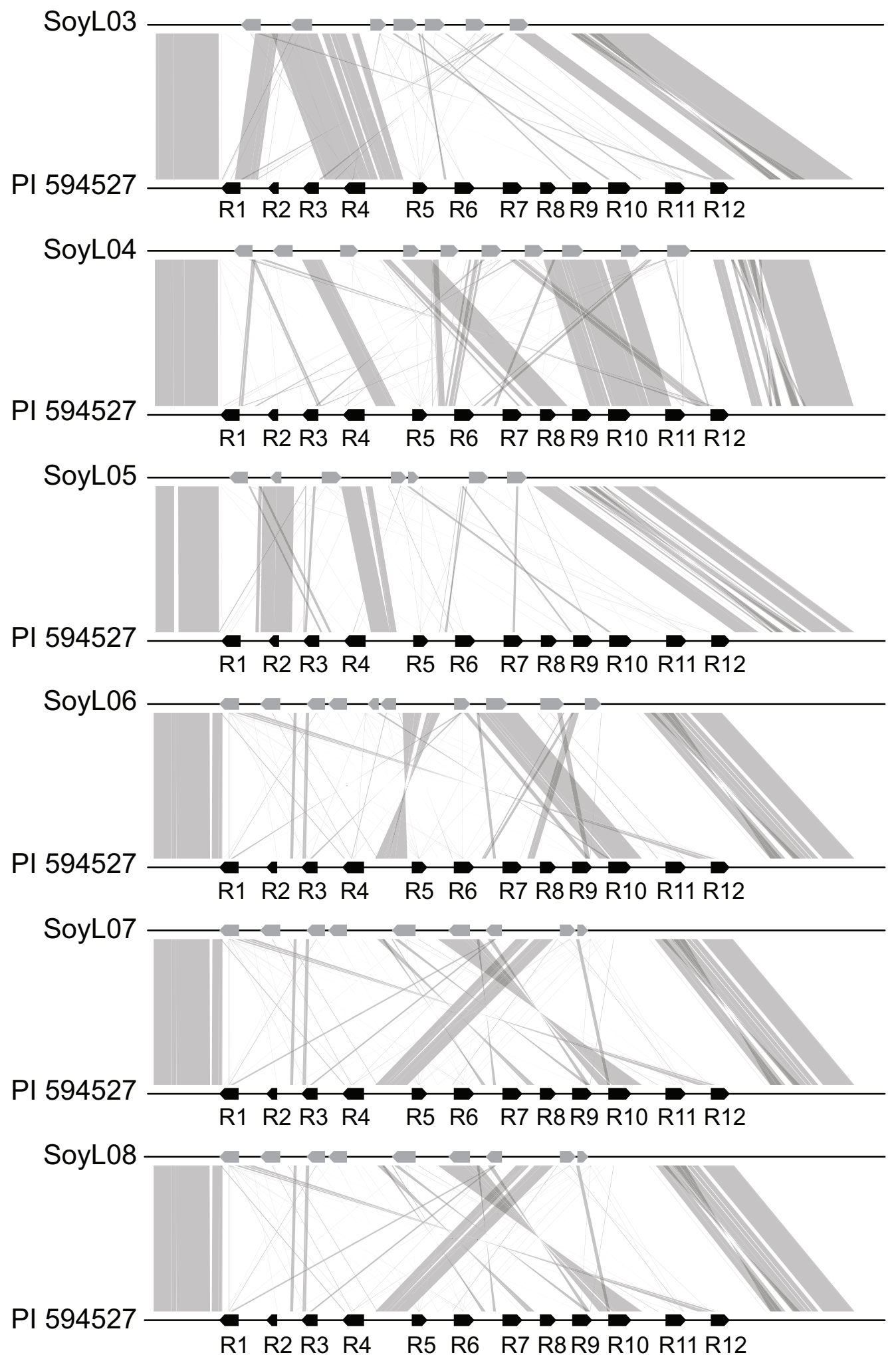

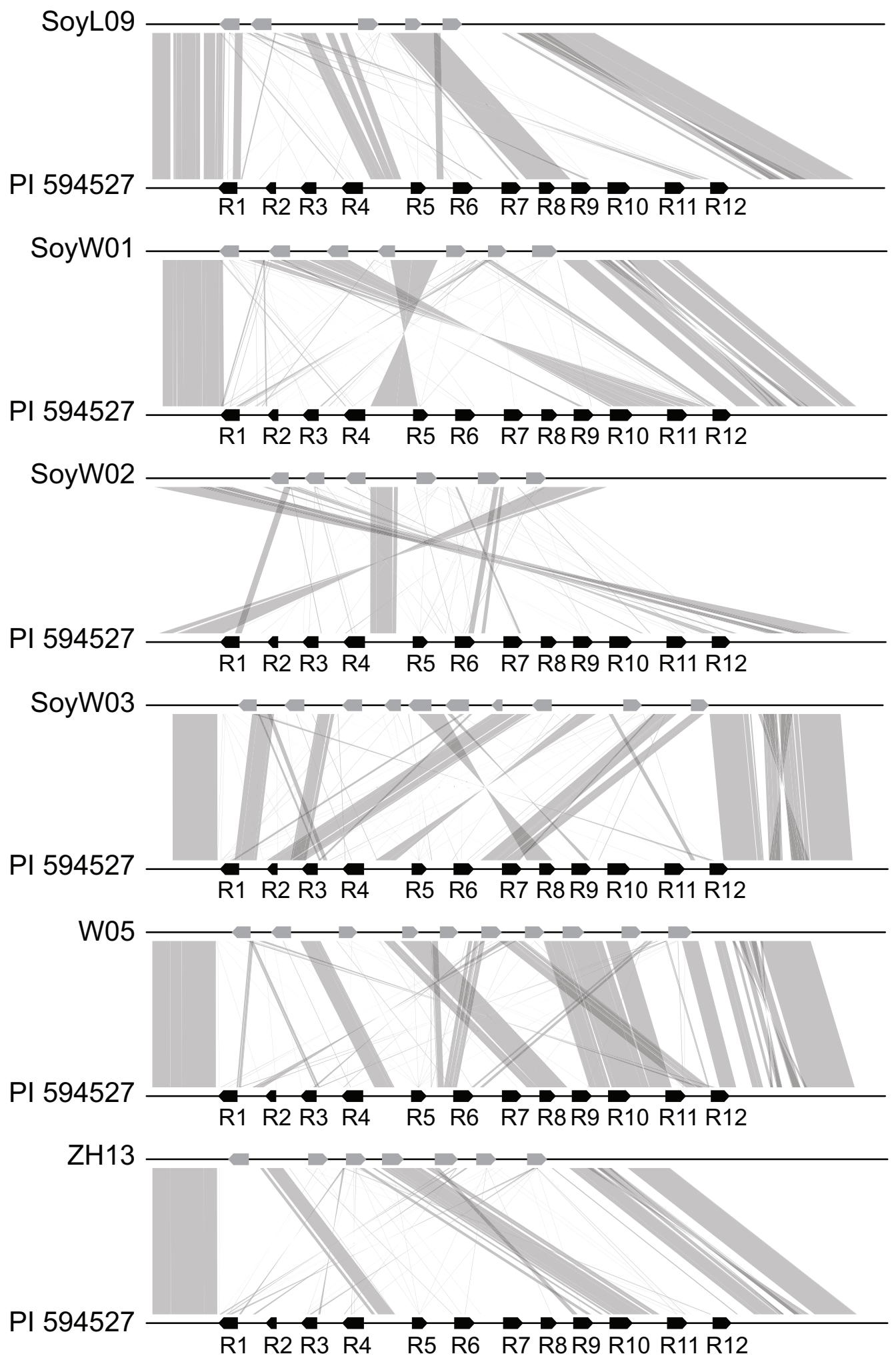

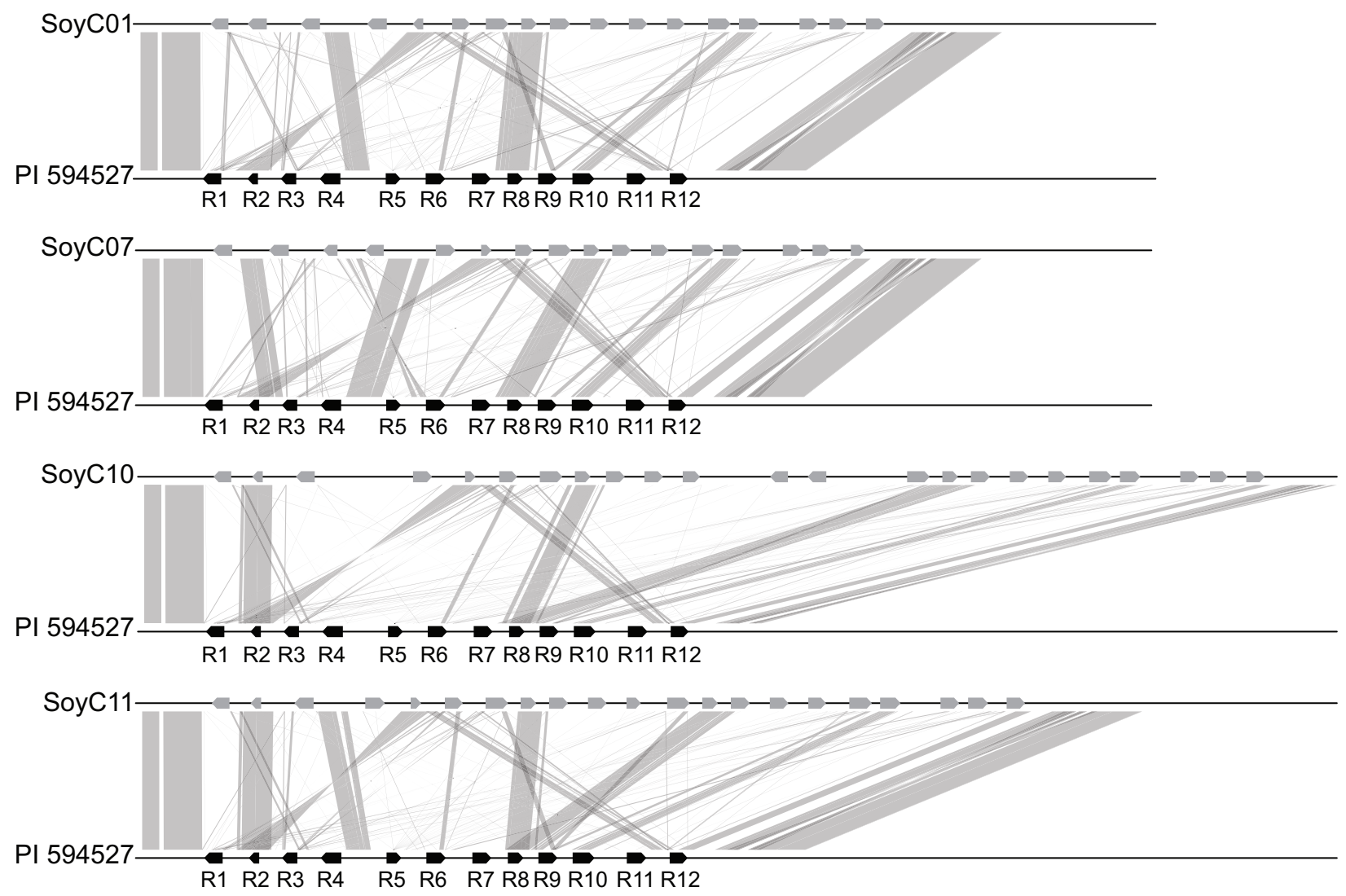

$521 \quad$ Extended Data Fig. 9 | Sequence comparisons between the Rps11 donor line and 29 diverse

522 soybean genomes in the Rps11 region. Black boxes represent NBS-LRR genes from PI 594527, 523 grey boxes represent NBS-LRR genes from other soybean genomes, and grey shade represent 524 homologous alignments between genomes. 


\section{Supplementary Files}

This is a list of supplementary files associated with this preprint. Click to download.

- WangSupplementallnformation.pdf

- nrreportingsummary.pdf 